\title{
The Landscape of Prescription Drug Shortages During the COVID-19 Pandemic
}

\author{
Erin R. Fox ${ }^{1} \cdot$ Andrew I. Stolbach ${ }^{2} \cdot$ Maryann Mazer-Amirshahi ${ }^{3,4}$ \\ Received: 13 May 2020 / Revised: 13 May 2020 / Accepted: 15 May 2020 / Published online: 27 May 2020 \\ (C) American College of Medical Toxicology 2020
}

Keywords Prescription drug shortages · COVID-19 pandemic

\section{Background}

Prescription drug shortages have created clinical and patient safety challenges for many years, affecting some of the most basic medical products $[1,2]$. Historically, the majority of drug shortages have impacted generic injectable products and were attributable to manufacturing facility quality problems and a lack of supply chain redundancy. In 2014, experts from the FDA noted that "to truly eradicate or minimize the occurrence of supply interruptions and or drug shortages, manufacturers must not only comply with good manufacturing practices, but also must invest in modernizing and or improving manufacturing quality systems" [3]. Unfortunately, successful long-term solutions have yet to be put in place.[3, 4] An interagency drug shortage task force established in 2018 made number of recommendations, including a rating system to incentivize improvements in manufacturing quality management systems that have yet to be implemented [4]. While there has been some improvement in manufacturer notifications, allowing the FDA to prevent some drug shortages, the USA supply chain was experiencing shortages in various analgesics, sedatives, cardiovascular drugs, and antimicrobials in early 2020 based on both the FDA and American Society of Health-System Pharmacists drug shortage databases [5, 6].

Supervising Editor: Mark B. Mycyk, MD

Maryann Mazer-Amirshahi

maryannmazer@gmail.com

1 Department of Pharmacy, University of Utah Health, Salt Lake City, UT, USA

2 Johns Hopkins School of Medicine, Baltimore, MD, USA

3 Department of Emergency Medicine, MedStar Washington Hospital Center, 110 Irving St NW, Washington, DC 20010, USA

4 Georgetown University School of Medicine, Washington, DC, USA

\section{The Shortage Landscape During COVID-19}

The COVID-19 pandemic struck the USA at a time when the drug supply was already vulnerable, resulting in drug shortages that have differed from prior episodes in several key ways. Unlike prior drug shortages caused mainly by supplyside issues, this one is principally a problem of demand. The sudden spike in critically ill patients requiring intensive medical care has depleted the supply of many medications and products - particularly, generic parenteral sedatives and analgesics. Manufacturers, who customarily maintain inventories to satisfy only a few months of typical need, simply do not have the resources to meet this unprecedented demand.

Manufactures face a variety of challenges in increasing production. Most manufacturers of generic injectable products do not have additional capacity or open manufacturing lines that can be repurposed [7]. Manufacturing of sterile products is a delicate process performed in a limited number of facilities due to high operating costs. Bringing a new manufacturing facility into operation often requires years. Repurposing an existing production line to produce drugs that are in high demand may precipitate critical shortages of other medications [7]. Manufacturers of controlled substances, including sedatives and analgesics, face additional challenges as annual production quotas are tightly controlled by the Drug Enforcement Agency (DEA). Due to increased demand, on March 31, 2020, multiple healthcare organizations requested that the DEA increases production quotas [8]. Although DEA increased quotas for manufacturing of controlled substances on April 17, 2020, the ability to upscale the manufacturing process is limited due to the aforementioned manufacturing issues. In the past, the USA has turned to importation of drugs from other countries but this is not feasible now given the global nature of the pandemic.

Prior to the COVID-19 pandemic, shortages of medications mainly involved injectable products. Now, we 
are seeing shortages precipitated by increased demand for drugs that are popularly perceived as potential COVID-19 therapies, such as hydroxychloroquine and chloroquine. The recognition of the risks of viral aerosolization by nebulizer treatments has spiked demand for metered-dose-inhalers, resulting in critical shortages of these products, as well. Demand for chronic medications increased as patients rushed to refill prescriptions prior to "stay at home" orders. Finally, another key difference in the landscape of drug shortages in the time of COVID-19 is that many of the bedside work-arounds used to mitigate shortages in the past may put staff at risk of infection.

\section{Recommendations}

Others have written extensively on institutional frameworks for addressing drug shortages $[9,10]$ and ethically allocating drugs in limited supply [11]. When appropriate, medications may be substituted with second- and third-line agents, alternative strengths, and formulations. In rare cases, there may be no reasonable therapeutic equivalent. When this occurs, ethics experts in conjunction with pharmacy, medicine, and nursing leadership should be called together to create a patient prioritization scheme. In general, drug administration should be prioritized for patients receiving the drug for conditions where there is proven, life-saving benefit over those with in conditions where the drug is investigational.

New procedures create the opportunity for medication errors, delayed or suboptimal therapy, and adverse drug events. The use of unfamiliar medications may result in dosing and administration errors, particularly if the products are not fully built into electronic health records. Using unfamiliar formulations can result in incorrect dosing or improper dilution. Use of second- and third-line agents may predispose patients to adverse drug effects that do not occur with first line agents. For example, when lorazepam is substituted for midazolam in ventilated patients, the propylene glycol vehicle can cause a dose-limiting hyperlactatemia. Healthcare organizations must be proactive and vigilant. Ideally, as soon as staff learns about a drug shortage, education about new protocols should be conducted, and updates in electronic health record, information technology resources, and barcoding should already be in place.

Risks to staff may be minimized by bundling medication administration and other nursing care whenever possible, but procedures that preserve medications and supplies may be at odds with protecting staff safety. The use of albuterol nebulizers protects the supply of metered-dose inhalers but creates potential for viral aerosolization. Some institutions have developed common-canister protocols to help conserve inhalers
[12]. Keeping medication pumps outside of rooms may preserve scarce PPE and minimize staff exposure to infected patients-at the cost of additional IV sets. For a patient who only requires brief sedation, fentanyl bolus dosing may preserve drug, pumps, and IV sets, but may require more bedside exposure to infected patients and PPE use. If medication routes are changed from infusions to intravenous push, make sure specific instruction are provided to nursing staff regarding the need to dilute and administration time [13].

Communication at all levels is essential. When the central pharmacy foresees an impending shortage, clinical pharmacists and pharmacy and therapeutics decision makers should be given ample opportunity to prepare plans for alternative agents. As elective surgeries resume, coordination between service lines can ensure a safety stock of essential PPE, and medications are available for a sudden surge of COVID-19 patients (such as an outbreak from a skilled nursing facility) [14]. When a second- or third-line agent is used because of a shortage situation, the rationale for its use should be documented in the medical record. As the pandemic continues to evolve, institutions must reevaluate their current shortage framework and modify as necessary, and at the same time, communicating changes to front-line providers.

\section{Summary}

The COVID-19 pandemic has created a new type of drug shortage-one caused by increased demand. Supplies of generic injectable sedatives and analgesics are stretched thin by the sudden spike in critically ill patients. Decision makers must develop protocols for new formulations of existing drugs and administration of second- and third-line drugs. Inevitably, unfamiliar procedures create opportunity for medication errors and adverse drug events. Robust information technology resources will be required to support these protocols. All procedures should consider the health and safety and staff by minimizing bedside exposure and need for PPE.

Funding Information No funding was provided for this study.

\section{Compliance with Ethical Standards}

Conflicts of Interest None.

\section{References}

1. Mazer-Amirshahi M, Fox ER. Saline shortages - many causes, no simple solution. N Engl J Med. 2018;378(16):1472-4.

2. Mazer-Amirshahi M, Goyal M, Umar SA, Fox ER, Zocchi M, Hawley KL, et al. U.S. drug shortages for medications used in adult critical care (2001-2016). J Crit Care. 2017;41:283-8. 
3. Dill S, Ahn J. Drug shortages in developed countries-reasons, therapeutic consequences, and handling. Eur J Clin Pharmacol. 2014;70(12):1405-12.

4. Food and Drug Administration. Drug shortages: root causes and potential solutions. October 2019, Updated February 2020. https://www.fda.gov/drugs/drug-shortages/report-drug-shortagesroot-causes-and-potential-solutions. Accessed 23 Apr 2020.

5. Food and Drug Administration. FDA drug shortages. https://www. accessdata.fda.gov/scripts/drugshortages/default.cfm. Accessed 4 May 2020

6. American Society of Health-System Pharmacists. Current drug shortages. https://www.ashp.org/Drug-Shortages/CurrentShortages. Accessed 4 May 2020.

7. Woodcock J, Wosinska M. Economic and technological drivers of generic sterile injectable drug shortages. Clin Pharmacol Ther. 2013;93(2):170-6.

8. Agency DE. DEA takes additional steps to allow increased production of controlled substances used in COVID-19 care. https://www. dea.gov/press-releases/2020/04/07/dea-takes-additional-stepsallow-increased-production-controlled Accessed 23 April 2020.

9. Fox ER, McLaughlin MM. ASHP guidelines on managing drug product shortages. Am J Health Syst Pharm. 2018;75(21):1742-50.
10. Hick JL, Hanfling D, Courtney B, Lurie N. Rationing salt waterdisaster planning and daily care delivery. N Engl J Med. 2014;370(17):1573-6.

11. Rosoff PM, Patel KR, Scates A, Rhea G, Bush PW, Govert JA. Coping with critical drug shortages: an ethical approach for allocating scarce resources in hospitals. Arch Intern Med. 2012:1-6.

12. Centers for disease control and prevention. Healthcare infection prevention and control FAQs for COVID-19. https://www.cdc. gov/coronavirus/2019-ncov/hcp/infection-control-faq.html Accessed 23 April 2020.

13. Institute for safe medication practices. Acute Care Special Edition, COVID-19. ISMP Medication Safety Alert! https://ismp.org/acutecare/special-edition-medication-safety-alert-april-9-2020/covid-19. Accessed 5 May 2020.

14. Joint Statement. Safely resuming elective surgery as COVID-19 curve flattens: ACS, ASA, APRN, and AHA roadmap for readiness. https://www.aha.org/press-releases/2020-04-17-safelyresuming-elective-surgery-covid-19-curve-flattens-acs-asa-aornand. Accessed 23 April 2020.

Publisher's Note Springer Nature remains neutral with regard to jurisdictional claims in published maps and institutional affiliations. 